\title{
Chromatic Dispersion of Thin Film Filters
}

\author{
R. M. Fortenberry, M. A. Scobey, D. J. Derickson, L. F. Stokes, P. C. Egerton \\ Bookham Inc. 3640 Westwind Blvd, Santa Rosa, California 95403 \\ Mike.Scobel@Bookham.com
}

\begin{abstract}
This paper discusses the chromatic dispersion effects of thin film filters used in telecommunications systems. ITU channel, banded architecture, and low dispersion filter results are presented. Design techniques to improve and manage chromatic dispersion in practical network implementations are discussed.
\end{abstract}

\section{Introduction}

Optical thin film filters (TFFs) have been widely deployed in telecommunications optical networks for a number of applications including channel multiplexing and demultiplexing, optical add drop, banded architecture multiplexing and demultiplexing, and dichroic bandsplitting among others. The deployment of thin film filters has been driven by their highly desirable attributes such as ease of manufacture, thermal stability, and flat, square filter spectral profiles. In most cases the chromatic dispersion (CD) inherent in TFFs is not a significant concern; however, as bit rates and optical channel densities continue to increase, the issue of CD in TFFs has grown in importance. This paper discusses chromatic dispersion effects of TFFs both in theory and as implemented in modern networks.

\section{Thin film filter background}

Thin film filters (TFFs) are typically designed as a set of stacked Fabry-Perot cavities with the mirrors consisting of a stack of high and low index quarterwave dielectric layers [1]. The reflectivity of the mirrors of each cavity, the properties of the spacers between the mirrors, and the number of cavities determine the optical properties such as the bandwidth and spectral slopes. With narrowband ITU channel filters, which are typically used to multiplex (mux) or demultiplex (demux) individual channels, the group delay (GD) peaks near the filter $3 \mathrm{~dB}$ points and gives a characteristic group delay as shown in Fig. 1 with a shape resembling "bat ears". For this filter type, CD grows rapidly as channel density increases. For a $50 \mathrm{GHz}$ ITU channel drop filter, group delay variation from peak to valley is twice as large as that of a $100 \mathrm{GHz}$ filter of similar design. Further, the $50 \mathrm{GHz}$ filter transmission band is half as wide as that of the $100 \mathrm{GHz}$ filter, with the group delay peak half as far from the filter center. Thus the maximum value of $\mathrm{CD}$, the derivative of group delay with respect to wavelength, is approximately 4 times higher for the $50 \mathrm{GHz}$ filter compared to the $100 \mathrm{GHz}$ filter (with identical adjacent channel isolation). Similarly, the maximum value of $\mathrm{CD}$ for a $25 \mathrm{GHz}$ filter is roughly 16 times that of the $100 \mathrm{GHz}$ filter [2].

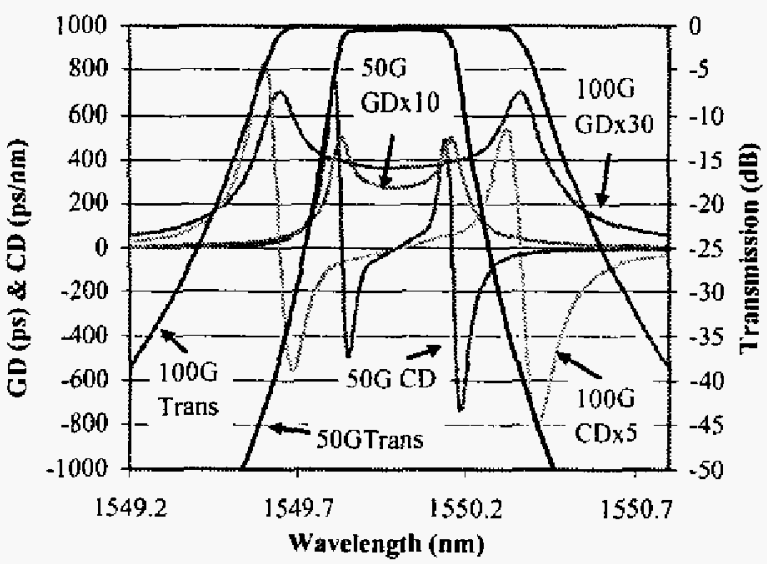

Fig. 1. $50 \mathrm{GHz}$ and $100 \mathrm{GHz} \mathrm{GD}$ and $\mathrm{CD}$ plots.

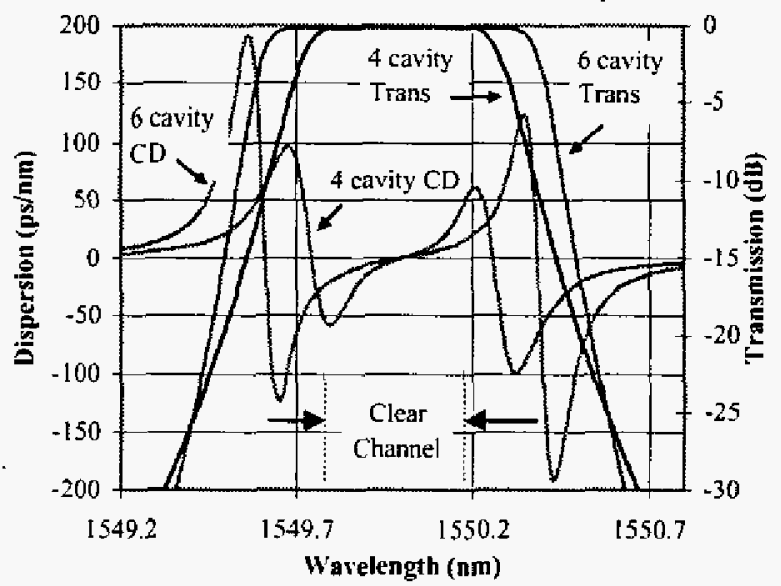

Fig. 2. Effect of cavity count on CD for $100 \mathrm{GHz}$ flat top

The optical performance of a thin film filter can be mathematically described as an infinite impulse response (IIR) filter [3]. In transmission a TFF is also a minimum phase filter and thus the transmission amplitude response of the filter is directly related to the phase response via a Hilbert transform or the equivalent Kramers-Kronig 
relationship. It follows that the CD of a bandpass TFF is unambiguously determined from the transmission properties. Another outcome of the relationship is a useful "rule of thumb" that the peak value of group delay is directly proportional to the rate of change of the slope of the transmission.

Since the relationship between phase and amplitude is a Hilbert transform, it is possible improve the $C D$ within the passband while minimizing the effect on the amplitude over that same region by making the changes outside of the central passband region. Increasing the cavity count of the filter design allows the $3 \mathrm{~dB}$ bandwidth to increase while maintaining transmission isolation, reducing inband $C D$ as shown in Fig. 2. Another approach to reducing $C D$ is to slightly modify the transmission profile as shown in Fig. 3. The flatness of the group delay results from the rounded transmission profile in the center of the filter $[4,5]$. If the flatness of the amplitude response of a bandpass TFF is not critical, then the sharp spectral band edges of the filter can be reduced to the point of completely eliminating the group delay "bat ears" and the resultant filter has flat group delay, with near zero CD as shown in Fig. 4. Filter rounding can be even further increased beyond this point of minimal $\mathrm{CD}$ to create a filter with $\mathrm{CD}$ slope opposite to that of a flat top design.

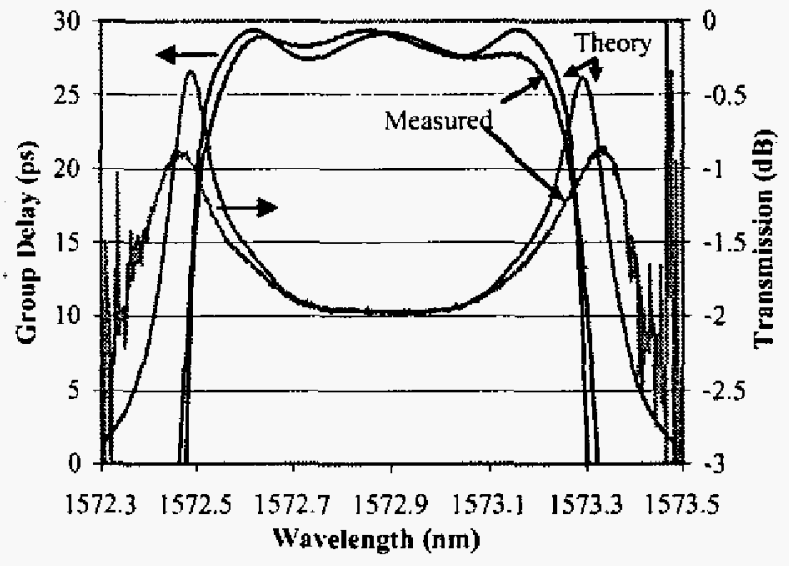

Fig. 3. 100GHz filter designed for low dispersion using a rouncied amplitude response.

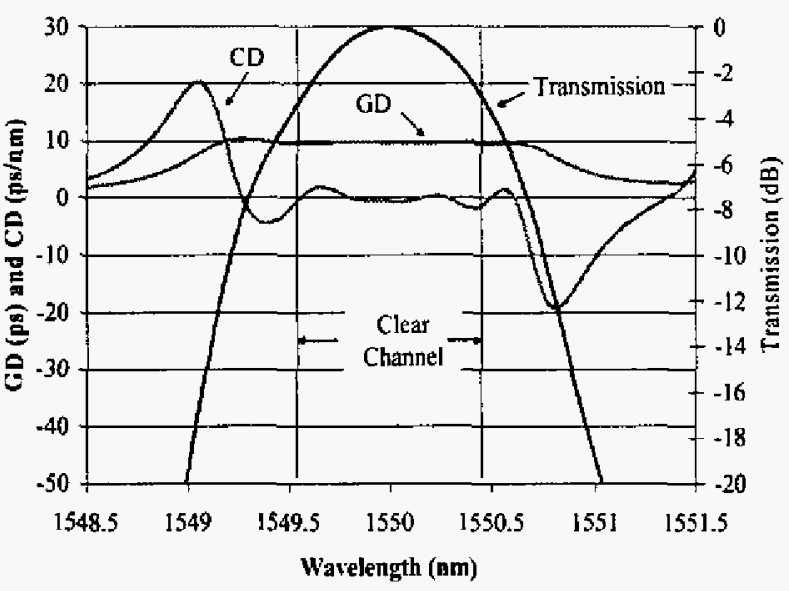

Fig. 4. $100 \mathrm{GHz}$ rounded top fiter design with minimal $\mathrm{CD}$.

It is worth noting that the peak value of CD of a TFF over the passband cannot be compared directly to the CD value of optical fiber because the filter $C D$ is not constant in wavelength. The group delay is symmetric about the filter center wavelength and so negative and positive sidebands of the optical carrier centered on the filter see the same (positive valued) group delay. In a length of fiber, these sidebands see opposite signed group delay relative to the carrier. The latter causes more distortion than the former. The result is that the dispersion power penalty of a narrow bandpass TTF is less than that of an equivalent fiber of the same peak CD value. Accurate analysis requires numerical simulations to obtain a prediction of its effect in a given transmission system.

The previous discussion has focused on the transmission properties of a TFF. With symmetric TFF designs (i.e. where the design is the same when reversing the order of the layers) the reflection is also minimum phase and the Hilbert transform relationship applies to the reflected light as well. If the filter is asymmetric then the relationship between phase and amplitude is not restricted by the Hilbert transform and the designer has, in general, much more freedom to change the $\mathrm{CD}$ relative to the amplitude. This is demonstrated in particular with Gires-Tournois (GT) interferometer thin film devices such as is discussed in section 6 .

\section{Banded architecture (Skip filters)}

TFF "skip" filters split, mux/demux, or add and drop continuous bands of channels. They are popular for their "payas-you-grow" nature, providing a simple and inexpensive method of increasing capacity by adding groups of channels to previously installed links. Fig. 5 shows a measured example of a 10 skip 0 filter for $100 \mathrm{GHz}$ channel spacing [6]. The "10 skip 0" designation means that 10 channels can pass in transmission through the filter and all other ITU channels are completely reflected. CD of these types of filters is identical to those discussed above except that the channels are now a small fraction of the filter width. Channels can reside on either the edge of the filter where the $\mathrm{CD}$ is largest or at the center where the $\mathrm{CD}$ is zero. Channels on opposite edges have opposite CD slopes. 
In contrast, a 4 skip 1 filter would pass 4 channels in transmission but the channel at the filter skirt is partially transmitted and partially reflected causing the channel to be lost (or "skipped").
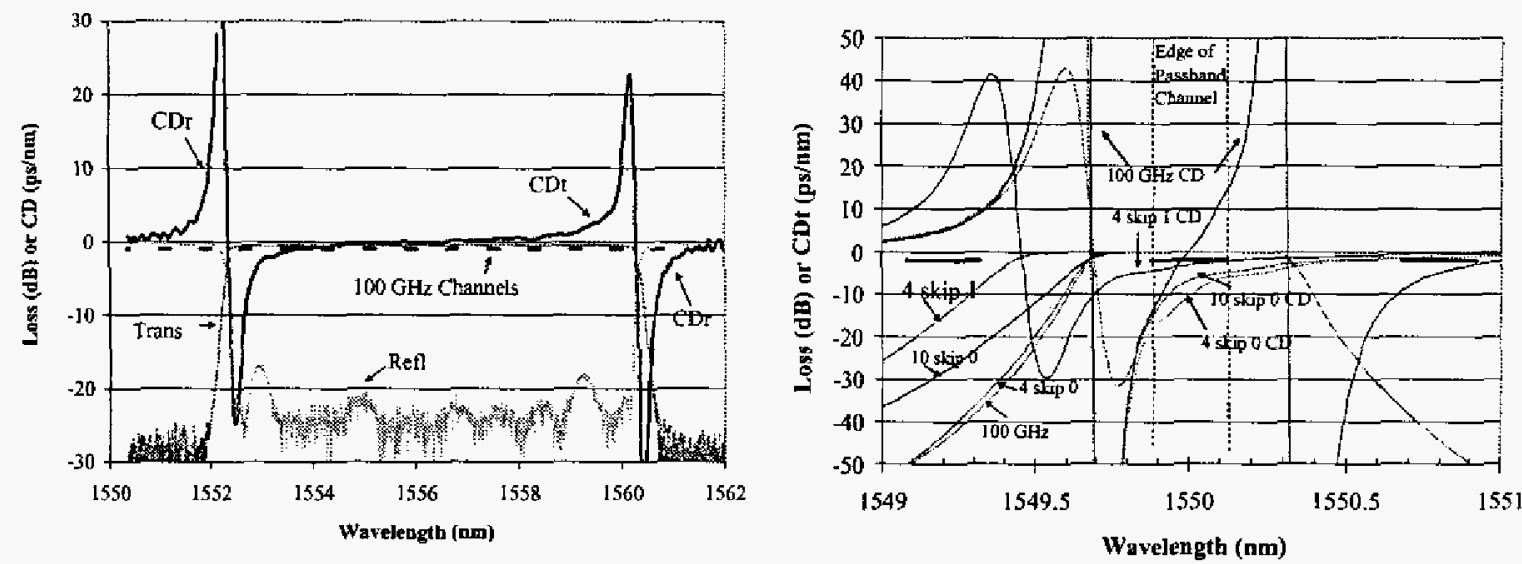

Fig. 5. Measured CD in Reflection (CDr) and Transmission (CDt) of 10s0

Fig. 6. Calculated loss and $\mathrm{CDt}$ of various banded filters.

For practical applications, how does the CD of a skip filter compare to that of a narrowband filter? Fig. 5 shows a comparison of $100 \mathrm{GHz}$ versions of a 10 skip 0,4 skjp 0 , and 4 skip 1 as well as a $100 \mathrm{GHz}$ single channel filter with all of the filters lined up at a channel at the edge of the passband In this figure, the $100 \mathrm{GHz}$ channel filter and the 4 skip 0 filter are designed for $25 \mathrm{~dB}$ adjacent channel isolation and flat top passbands. The $\mathrm{CD}$ in transmission for both the $100 \mathrm{GHz}$ channel filter and the 4 skip 0 are very similar at the edge of the filter passbands. The steepness of the 4 skip 1 and the 10 skip 0 functions are similar leading to similar shapes of the CD. The 4 skip 1 filter however has a lower effective $C D$ for its outer channel, because the outer channel is farther from the filter edge due to the skipped channel.

\section{Packaged filter considerations}

The optical performance of a packaged TFF is affected by a number of factors including beam divergence, thermal stability, wavelength centering, among others. Wavelength centering is particularly important as error in centering the filter during the packaging process increases $\mathrm{CD}$ since the $\mathrm{CD}$ is lowest at the center wavelength.

Figure 7 shows the $\mathrm{CD}$ of a TFF before and after packaging. The transmission and reflection spectral profiles are somewhat rounded in the packaged filter case. The passband is narrower and the peak $C D$ is lower after packaging due to filter rounding caused by beam divergence as well as other packaging issues.
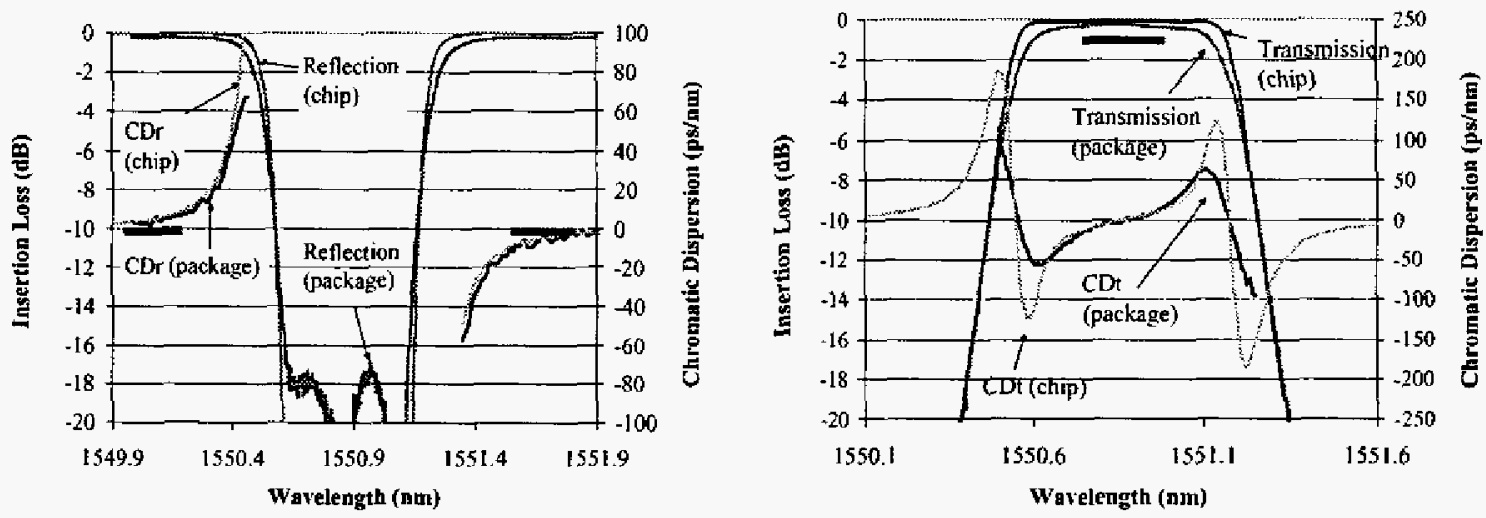

Fig. 7. Amplitude and CD of TFF filter before (chip) and after packaging for reflection in chart on left and transmission on the right.

\section{Module Considerations}

Modules are constructed by cascading TFFs in a three port package via splicing. A three port package aligns the input/common, reflection and transmission signals to individual optical fibers. In a module cascade, the worst case channel $\mathrm{CD}$ is the sum of the transmission $\mathrm{CD}$ and the adjacent channel reflection $\mathrm{CD}$. Figure 8 show two module 
architectures, the first being a simple cascade and the second using a $3 \mathrm{~dB}$ coupler to create two legs of interleaved channels. The interleaved channels reduce total $\mathrm{CD}$ by increasing the adjacent channel spacing (which reduces reflection $\mathrm{CD}$ ). The $3 \mathrm{~dB}$ coupler trades off increased loss for lower $\mathrm{CD}$ in this design.

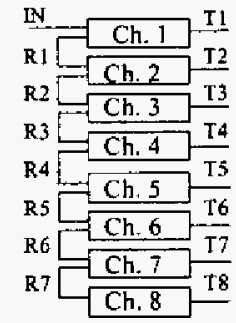

(a)

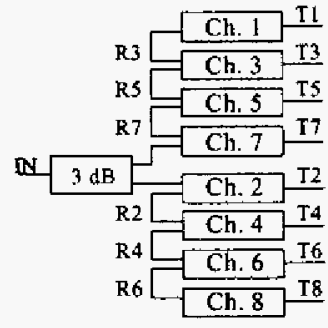

(b)

Fig. 8. Module architectures for TFF. $8 \mathrm{a}$ is a simple cascade. $8 \mathrm{~b}$ trades off loss for lower CD.

\section{CD compensation using a Gires-Tournois (GT) etalon}

The characteristic CD of a flat top TFF can be compensated by a GT etalon designed to have opposite slope and equal magnitude of $C D$ to the TFF filter. An example of this approach is shown in Fig. 9, where a GT compensator filter was fabricated using a single cavity TFF and spliced in series with the transmission signal of a $50 \mathrm{GHz}$ TFF filter. As can be seen in the figure, the $C D$ has been reduced across the passband, and nearly eliminated in the center 100 picometers.
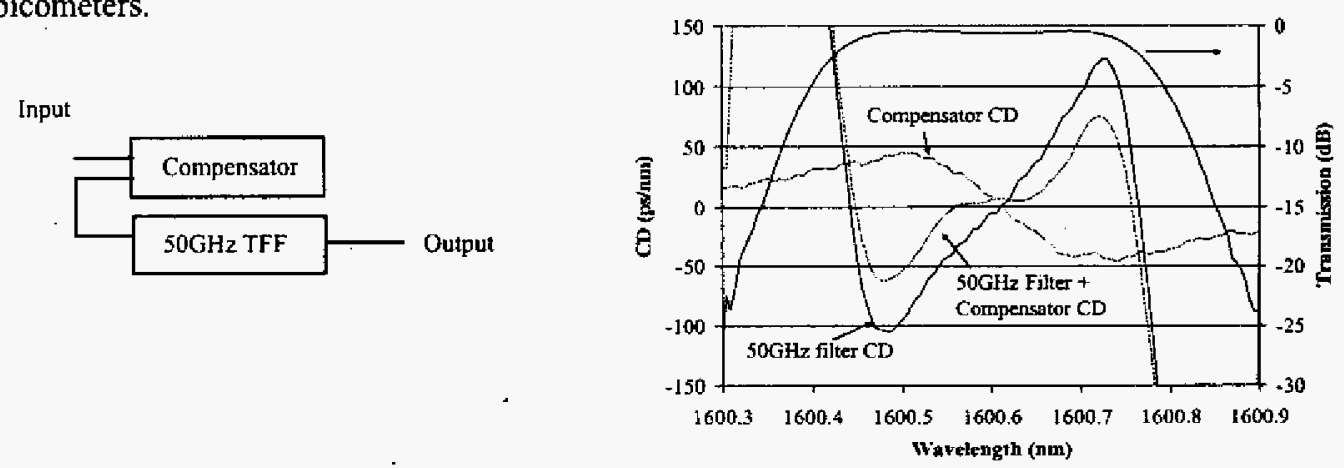

Fig. 9. Chromatic dispersion of $50 \mathrm{GHz}$ filter, GT compensator, and combination of both as shown in schematic on left.

\section{Summary}

Thin film filters have a wide variety of applications in optical communications due to their outstanding spectral performance and low cost. TFFs have inherent chromatic dispersion in both transmission and reflection that can be managed in the design process. Design techniques to lower CD include adjustment of cavity count, varying the passband amplitude shape, and cascading with compensation elements. CD of TFF is also unique relative to fiber as it is zero at the cènter of the passband, which provides lower inherent dispersion power penalties for a given $C D$ value. CD of TFFs at a module level can be managed by the use of module architecture designs which tradeoff insertion loss for lower $\mathrm{CD}$.

\section{Acknowledgements}

The authors wish to thank Victoria Crossman and Rad Sommer for their contributions and insight.

\section{References}

[1] H. A. Macleod, Thin Film Optical Fillers (Institute of Physics Publishing, Bristol, 2001), Chap. 7.

[2] M. A. Scobey, R. M. Fortenberry, L.F Stokes, W. P. Kastanis, D. J. Derickson, "Thin film interference filters for $25 \mathrm{GHz}$ channel spacing," in Technical Digest Optical Fiber Communications (OFC) Conference 2002, paper ThC5, page 398.

[3] C. K. Madsen and J. H. Zhao, Optical Filter Design and Analysis (John Wiley and Sons, New York, 1999), Chap. 3.

[4] R. M. Fortenberry, M. E. Wescott, L. P. Ghislain and M. A. Scobey, "Low Chromatic Dispersion Thin Film DWDM Filters for 40 Gb/s Transmission Systems," in Technical Digest Optical Fiber Communication (OFC) Conference 2002, paper WS2, page 319.

[5] R. M. Fortenberry, D. Derickson, and M. A. Scobey, "Optical Filter Dispersion in 40 Gb/s DWDM Transmission Systems," in Technical Proceedings National Fiber Optic Engineers Conference (NFOEC) 2001, page 670.

[6] Dennis Derickson, Rance Fortenberry, Mike Scobey, Rad Sommer and Loren Stokes, "Advancements in thin film-fillers for telecommunications applications" SPIE IT-COM, Orlando Florida, Sept. 2003 (lnvited Paper). 